\title{
CFD Modeling of Rotating Spiral Wound Membrane with Corrugated Spacer: Shear Rate Effect
}

\author{
Abdul Latif Ahmad, Ban Zhen Hong and Ooi Boon Seng \\ School of Chemical Engineering, Engineering Campus, Seri Ampangan, Universiti Sains Malaysia, Nibong Tebal, Pulau Pinang \\ 14300, Malaysia
}

Received: August 13, 2010 / Accepted: August 27, 2010 / Published: February 10, 2011.

\begin{abstract}
Shear rate is proven in many studies to be playing an important role in reducing the concentration polarization and membrane fouling. This paper investigates the effect of rotation speed of corrugated spacer on the shear rate using computational fluid dynamics (CFD). Two small channels with the inner membrane (IM) $39 \mathrm{~mm}$ from the axis of rotation and outer membrane (OM) with $41.5 \mathrm{~mm}$ were used for the studies. The inlet pressure is set as $100 \mathrm{kPa}$ and water is used to study the shear stress on the rotating membrane. The average surface shear rate between the two membranes is found out to be increasing with the rotation. The average shear rate on stationary membrane is $25,645 \mathrm{~s}^{-1}$ and increased to $46,961 \mathrm{~s}^{-1}$ at rotation speed of $250 \mathrm{rad} / \mathrm{s}$. The shear rate of OM is larger than IM when the rotation speed is lower than $75 \mathrm{rad} / \mathrm{s}$ but the shear rate on IM is larger when rotation speed is higher than $75 \mathrm{rad} / \mathrm{s}$. The rotation of corrugated spacer can be applied to the spiral wound membrane module to increase the shear rate and thus increase the permeate flux and reduce the concentration polarization.
\end{abstract}

Key words: Shear rate, dynamic membrane, rotation.

\section{Introduction}

Shear rate is one of the factors determining the permeate flux in the membrane filtration process. Concentration polarization in ultrafiltration (UF), nanofiltration (NF) and reverse osmosis (RO) and also membrane fouling in microfiltration (MF) has been proven can be reduced by high shear rate [1]. The back transport of macromolecules or solute that concentrated on the membrane surface can be improved by high membrane surface shear rate [2] and thus prevent concentration polarization [3] and cake layer buildup [4]. Dynamic membrane filtration system has been introduced to increase the membrane surface shear rate by moving parts of the membrane system such as rotating disk filtration and vibration shear enhanced process (VSEP) [5]. The shear rate can be

Corresponding author: Abdul Latif Ahmad (1967- ), male, Ph.D., professor, research fields: membrane technology, wastewater treatment and separation processes. E-mail: chlatif@eng.usm.my. increased by decoupling the mechanical energy supplied and also the feed flow velocity [6]. The high shear rate is able to extend the pressure limited regime and thus the permeate flux can be increased with increase of transmembrane pressure (TMP) [7]. The permeate flux in dynamic membrane is found out to be a function of shear rate [8]. Thus, at the same TMP, the permeate flux is also higher in dynamic membrane compared to conventional crossflow [9] and tubular membrane [10].

Computational fluid dynamics (CFD) is a powerful tool for modeling and estimating the shear stress created on the membrane surface in dynamic membrane filtration. In this study, CFD software (FLUENT v6) will be utilized to study the effect of rotating the spiral wound membrane module on the shear rate. There are some assumptions made in this simulation as shown below:

(1) The permeate flux is very small compared to the fluid flow velocity, thus the hydrodynamics of the flow 
will not be affected by the permeate flux;

(2) The fluid flow adjacent to the wall is laminar.

\section{Boundary Condition}

FLUENT v6 was used for modeling and simulation in current work. In present simulation, pure water was used to study the flow characteristics. The water properties were defined in the software itself. The inlet gauge pressure was set as $100 \mathrm{kPa}$ for all simulation and the membrane was treated as impermeable wall. The outlet pressure from the small channel was not set. The simulations were performed using unsteady state laminar flow at ambient pressure. Dynamic mesh function was used to define the dynamic motion of the mesh where the motions were defined using user defined function (UDF). The UDF was written in ' $C$ ' programming language using predefined macro in the FLUENT solver. Macro 'Define_CG_Motion' for dynamic mesh was used in the UDF. The discretization of the governing equations was performed using a segregated incompressible flow solver in which each governing equation is solved separately. The velocity and pressure parameters would be linked and solved by SIMPLE algorithm. In order to achieve higher order of accuracy, second order upwind discretization schemes was selected to compute the momentum.

\section{Simulation Domain}

The computational domain is shown in Fig. 1. The simulated domain was 1 meter in length. The mesh size generated on the membrane surface is $8 \times 10^{-5} \mathrm{~m}$ between each node. The distance of the inner membrane (IM) with the axis of rotation is $39 \mathrm{~mm}$ and the outer membrane (OM) is $41.5 \mathrm{~mm}$. The $\mathrm{x}$ and $\mathrm{y}$ directions were the rotation direction and $\mathrm{z}$ direction was the direction of fluid flow from inlet to outlet. The motion track of the small channel is shown in Fig. 1c.

\section{Results and Discussion}

The shear rate of the surface was solved by fluent using Navier-Stoke equations for each rotating speed.

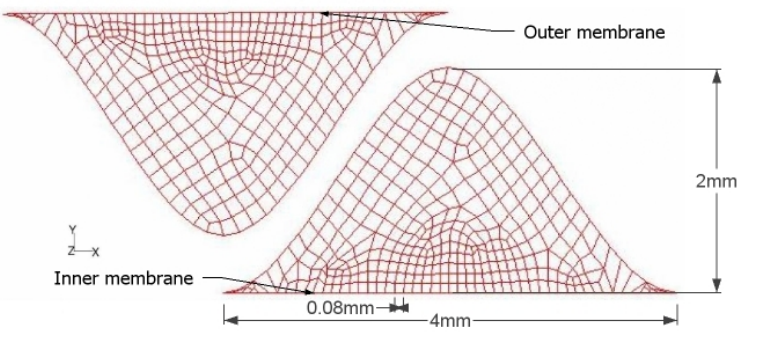

(a)

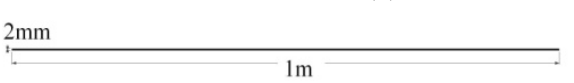

(b)

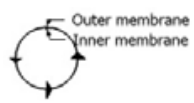

(c)
Fig. 1 Mesh and motion track of the small channel in the spacer, (a) front view (b) side view (c) motion track node.

The simulation results are divided into two parts; one was with lower rotation speed (2, 4, 8, 16, 24, 32, 40 $\mathrm{rad} / \mathrm{s}$ ) and another with higher rotation speed (50, 75, $100,125,150,175,200,225,250 \mathrm{rad} / \mathrm{s})$. Time step of $0.00005 \mathrm{~s}$ and the computational time of $1 \mathrm{~s}$ were used for all simulations. All the results shown in Fig. 2 were taken after the pressure drop and average shear rate are in steady state. In all the cases simulated, the flow achieved steady pressure drop and average shear rate within $0.5 \mathrm{~s}$.

\subsection{Effect of Rotational Speed on Membrane Shear Rate}

The average area weighted surface shear rate was computed from the average surface shear rate of inner and outer membrane. Fig. 2 shows the average surface wall shear rate versus the rotation speed of the small channel. The trend of the average shear rate on the membrane affected by rotation speed was different for low rotation speed and high rotation speed. The average shear rate increases steadily with the rotation speed beyond rotation speed of $50 \mathrm{rad} / \mathrm{s}$ but it changes exponentially at low rotation speed. No significant change in average shear rate at rotation speed below 16 $\mathrm{rad} / \mathrm{s}$ as shown in Fig. 2a. The average shear rate started to increase significantly when the rotation speed beyond $16 \mathrm{rad} / \mathrm{s}$. In overall, the average shear rate increases with rotation speed. This trend was formed due to the increase of fluid velocity adjacent to the membrane as the rotation speed increases. 


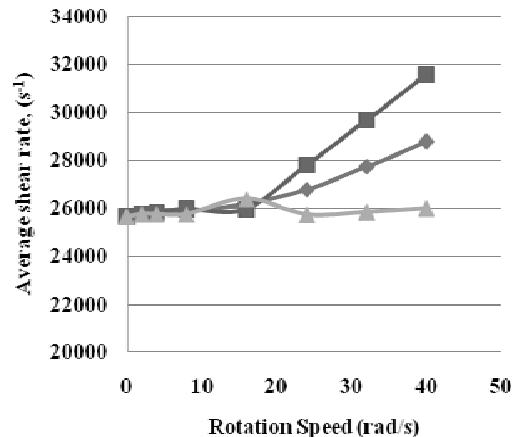

(a)

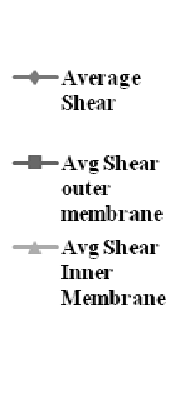

Fig. 2 Graph of average shear rate against rotation speed,

There were three forces involved applied on the fluid during rotation as shown in Fig. 3a. The pressure force was supplied by the fluid flow velocity, the centrifugal force was created due to the rotation and the tangential force was created by the spacer wall under no slip condition during rotation. The shear rate on $\mathrm{OM}$ was increased from stationary to rotation speed of $250 \mathrm{rad} / \mathrm{s}$ except at rotation speed of 16 and $75 \mathrm{rad} / \mathrm{s}$ but the shear rate on IM was increasing very slightly in the range of rotation speed lower than $50 \mathrm{rad} / \mathrm{s}$ and increased significantly after $50 \mathrm{rad} / \mathrm{s}$ as shown in Fig. 2. At rotation speed lower than $16 \mathrm{rad} / \mathrm{s}$, the difference between IM and OM is very small. Centrifugal force did not affect the flow significantly. In the range of rotation speed of 16 to $50 \mathrm{rad} / \mathrm{s}$, the pressure force is much higher than the tangential force and thus the internal flow still flowing in straight path towards the outlet. This path was affected by the centrifugal force in the range of rotation speed of 16 to $50 \mathrm{rad} / \mathrm{s}$ because the fluid flow direction was not affected and it was being pushed away from the axis of rotation. The forces acting on the fluid is shown in Fig. 3b. As expected, the fluid with high velocity will shifted near to the OM but away from the IM. Hence, the shear rate on OM will increase.

\subsection{Shear Rate Drop}

However, an unexpected very small drop of shear rate on OM can increase on IM at rotation speed of 16 $\mathrm{rad} / \mathrm{s}$. This is the only point where the shear rate on $\mathrm{OM}$ was less than shear rate on IM in the range of rotation

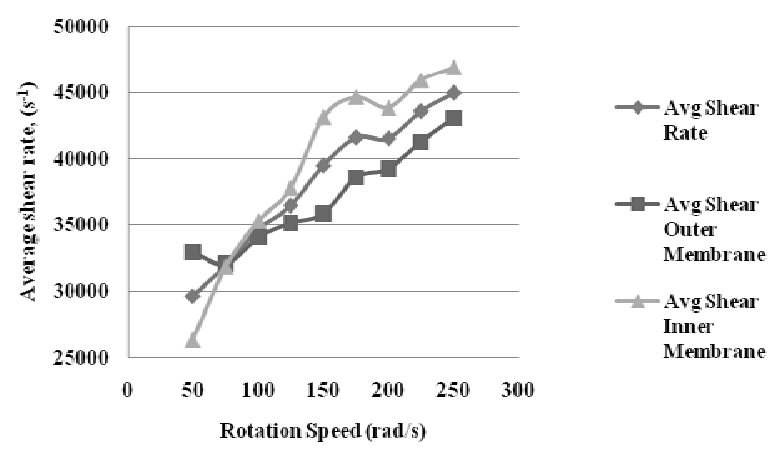

(b)

(a) low rotation speed (b) high rotation speed.

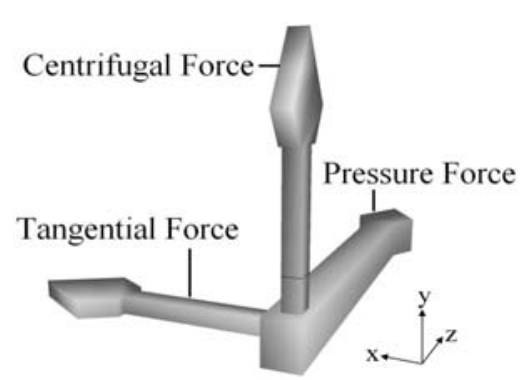

(a)

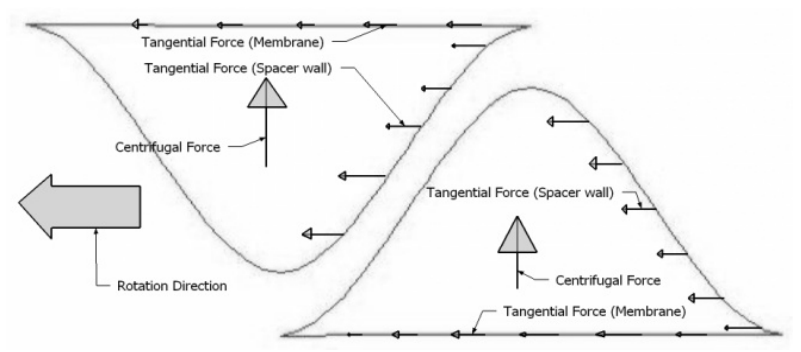

(b)

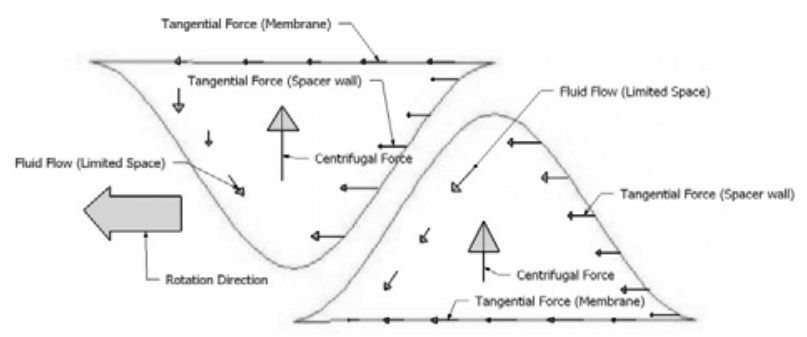

(c)

Fig. 3 Forces acting on the fluid flow in the outer and inner channel, (a) three forces acting direction (b) rotation speed lower than $75 \mathrm{rad} / \mathrm{s}$ (c) rotation speed higher than $75 \mathrm{rad} / \mathrm{s}$ due to limited space.

speed below $75 \mathrm{rad} / \mathrm{s}$. The drop of shear rate on OM was caused by the changes of flow direction of the fluid on the OM due to no slip wall condition. The velocity magnitude of the fluid on the OM increased more than the increase of fluid velocity magnitude adjacent to the 
OM. The increase of shear rate on IM was because the fluid velocity magnitude on the IM did not increase as much as fluid velocity magnitude adjacent to the IM. The difference of shear rate between IM and OM was because the distance travel by OM is larger than IM as the outer radius is larger than inner radius. Thus, the velocity magnitude on OM will be slightly larger than IM. This case only happens when the effect of centrifugal force was not significant.

There was another drop in shear rate on OM at rotation speed $75 \mathrm{rad} / \mathrm{s}$. This was the point where the fluid flow started to be limited by the geometry of the corrugated spacer and also the tangential force created by the spacer wall started to be dominant. The high tangential force in the outer channel pushed the fluid to flow in higher speed in $\mathrm{x}$ and $\mathrm{y}$ direction but due to the limited space, the fluid with high velocity will flow along the spacer wall towards the membrane. This flow will interrupt the flow adjacent to the OM which was created due to the tangential force created by spacer near OM combined with the centrifugal force. This interruption had caused a lower fluid flow velocity region adjacent to the $\mathrm{OM}$ as shown in Fig. 3c. Thus, the shear rate on the OM will decrease at rotation speed $75 \mathrm{rad} / \mathrm{s}$. The limited space effect due to the geometry of the spacer had affected both the flow in the outer and inner channel in different manner. The fluid will flow along the spacer of the inner channel and caused the fluid velocity to increase. In this case, the tangential force created by the spacer wall is not very high and thus the fluid flow did not interrupted as in outer channel.

\subsection{Effect of Rotational Speed on Inner and Outer Membrane Shear Rate}

The shear rate on OM becomes less than shear rate on IM when rotation speed was more than $75 \mathrm{rad} / \mathrm{s}$. This was partly because the tangential force was higher and the limited space effect had pushed the fluid back towards the axis of rotation. Another reason was because the fluid velocity on the OM was greater than the fluid velocity on IM due to the distance difference from axis. The fluid velocity on membrane will increase according to the rotation speed due to no slip condition. The fluid velocity adjacent to the membrane will increase due to the tangential force by the spacer wall. For the OM, the radius from the axis was higher and thus it had the higher tangential velocity than IM. The fluid velocity adjacent to the membrane increases in a larger magnitude due to the tangential force by the spacer wall. The shear rate on the OM less than IM because the increase of fluid velocity on the OM is greater than IM while they had similar fluid velocity adjacent to the membrane. This case was similar to the phenomenon seen at rotation speed at $16 \mathrm{rad} / \mathrm{s}$ but there were tangential and centrifugal forces affecting the fluid flow in this case. The velocity magnitude adjacent to the membrane increased due to these forces had directly increased the shear rate on the membrane.

\section{Conclusions}

The rotating corrugated spacer can create high shear rate during filtration process. This can overcome the problem of concentration polarization and cake layer formation in membrane filtration. The shear rate is governed by three forces which are pressure force, centrifugal force and tangential force due to the spacer wall. There is only pressure force affecting the fluid flow in the channel when the rotation speed is below 16 $\mathrm{rad} / \mathrm{s}$. The centrifugal force and pressure force are dominant when rotation speed of the channels in the range of rotation speed between 16 to $50 \mathrm{rad} / \mathrm{s}$. Tangential force due to the spacer wall starts to affect the flow when the rotation speed beyond $50 \mathrm{rad} / \mathrm{s}$. It has increased the resultant force that in turn increases the fluid velocity. The average shear rate starts to increase steadily when rotation speed beyond $75 \mathrm{rad} / \mathrm{s}$.

\section{Acknowledgments}

This work was supported in parts by Universiti Sains Malaysia Fellowship. 


\section{References}

[1] R. Bouzerar, P. Paullier, M.Y. Jaffrin, Concentration of mineral suspensions and industrial effluents using a rotating disk dynamic filtration module, Desalination 158 (2003) 79-85.

[2] R. Bian, K. Yamamoto, Y. Watanabe, The effect of shear rate on controlling the concentration polarization and membrane fouling, Desalination 131 (2000) 225-236.

[3] O. Akoum, M.Y. Jaffrin, L.H. Ding, Concentration of total milk proteins by high shear ultrafiltration in a vibrating membrane module, Journal of Membrane Science 247 (2005) 211-220.

[4] R. Bouzerar, L. Ding, M.Y. Jaffrin, Local permeate flux-shear-pressure relationships in a rotating disk microfiltration module: implications for global performance, Journal of Membrane Science 170 (2000) 127-141.

[5] M.Y. Jaffrin, Dynamic shear-enhanced membrane filtration: a review of rotating disks, rotating membranes and vibrating systems, Journal of Membrane Science 324 (2008) 7-25.

[6] S.P. Beier, G. Jonsson, Dynamic microfiltration with a vibrating hollow fiber membrane module, Desalination 199 (2006) 499-500.

[7] L.H. Ding, O. Akoum, A. Abraham, M.Y. Jaffrin, High shear skim milk ultrafiltration using rotating disk filtration systems, AIChE Journal 49 (2003) 2433-2441.

[8] M.D. Petala, A.I. Zouboulis, Vibratory shear enhanced processing membrane filtration applied for the removal of natural organic matter from surface waters, Journal of Membrane Science 269 (2006) 1-14.

[9] O. Akoum, M.Y. Jaffrin, L.H. Ding, M. Frappart, Treatment of dairy process waters using a vibrating filtration system and NF and RO membranes, Journal of Membrane Science 235 (2004) 111-122.

[10] O. Al-Akoum, L.H. Ding, M.Y. Jaffrin, Microfiltration and ultrafiltration of UHT skim milk with a vibrating membrane module, Separation and Purification Technology 28 (2002) 219-234. 